\title{
Effects of Scalar Dissipation Rate Fluctuations on Autoignition of Hydrogen/Air Mixture
}

\author{
Gaurav Bansal* and Hong G. $\mathrm{Im}^{\ddagger}$ \\ University of Michigan, Ann Arbor, Michigan 48109-2125 \\ and \\ Su-Ryong Lee \\ Seoul National University of Technology, Seoul 139-743, Republic of Korea \\ DOI: $\underline{10.2514 / 1.38665}$
}

\begin{abstract}
To understand the autoignition behavior in response to the flow turbulence, the effects of scalar dissipation rate fluctuation on the ignition of a nonpremixed hydrogen/air mixture is computationally studied using detailed chemistry in a counterflow configuration. A sinusoidal fluctuation of the scalar dissipation rate is imposed by oscillating the velocity at the nozzle inlet. Mean scalar dissipation rate is chosen to be close to the steady ignition limit, such that the instantaneous scalar dissipation rate can exceed the steady ignition limit during the oscillation. Response of the ignition delay to the frequency of the scalar dissipation rate oscillation is studied for two distinct cases, depending on whether the mean scalar dissipation rate at ignition kernel is less than (case $\mathrm{A}$ ) or greater than (case B) the steady ignition limit. For low frequencies, the ignition delay response for both cases is quasi-steady in that it correlates well with the mean scalar dissipation rate up to ignition delay. At high frequencies, however, the ignition delay response is significantly different for the two cases: for case $A$, the ignition delay increases with frequency and levels off at higher frequencies, whereas for case $B$, the ignition delay increases monotonically with frequency up to a critical value, beyond which no ignition is observed. A newly defined ignitability parameter is proposed based on the ignition-kernel Damköhler number such that all of the unsteady effects of scalar dissipation rate oscillation on ignition delay can be uniquely mapped to this parameter. Subsequently, a new criterion for ignitability is proposed based on this parameter.
\end{abstract}

\section{Nomenclature}

$A=$ amplitude of velocity fluctuation at the nozzle

$D a_{\mathrm{H}}=$ ignition-kernel Damköhler number, defined in Eq. (ㅁ)

$f \quad=$ frequency of velocity fluctuation at the nozzle

$t_{\mathrm{ign}}=$ ignition delay

$V_{0} \quad=$ initial velocity at the nozzle exit

$Z=$ mixture fraction

$\Gamma \quad=$ ignitability parameter defined in Eq. (8)

$\chi=$ scalar dissipation rate defined at the ignition kernel

$\bar{\chi}=$ mean scalar dissipation rate

$\chi_{s}^{*}=$ scalar dissipation rate at the steady ignition limit

\section{Introduction}

A UTOIGNITION of various fuel/air mixtures in a counterflow system has been a subject of extensive studies in the past. It is well known that autoignition occurs when the rate of radical generation by chemical branching, which may also be coupled with thermal feedback, exceeds the rate of radical loss by transport at the ignition kernel. The ratio of the two rates is characterized by the Damköhler number. A counterflow system allows a simplified and systematic model configuration in which the characteristic rates of reaction and transport can be independently controlled by adjusting the boundary conditions for composition, temperature, and

Presented as Paper 1005 at the 46th Aerospace Sciences Meeting and Exhibit, Reno, NV, 7-10 January 2008; received 20 May 2008; revision received 30 September 2008; accepted for publication 10 November 2008. Copyright $(\mathcal{2} 2008$ by Gaurav Bansal and Hong G. Im. Published by the American Institute of Aeronautics and Astronautics, Inc., with permission. Copies of this paper may be made for personal or internal use, on condition that the copier pay the $\$ 10.00$ per-copy fee to the Copyright Clearance Center, Inc., 222 Rosewood Drive, Danvers, MA 01923; include the code 0001-1452/ $09 \$ 10.00$ in correspondence with the CCC.

*Graduate Student, Department of Mechanical Engineering. Student Member AIAA.

${ }^{\dagger}$ Associate Professor, Department of Mechanical Engineering. Associate Fellow AIAA.

${ }^{\ddagger}$ Professor, Department of Automotive Engineering. velocities. In particular, the variation in the boundary velocities or, equivalently, in the strain rate translates to that in the scalar dissipation at the ignition kernel, which is considered to be an essential parameter that represents the effect of turbulence on autoignition. A number of previous studies [1-5] have reported detailed descriptions of the chemical and thermal structures of ignition kernels and have identified the ignition limits for hydrogen and hydrocarbon fuels under a wide range of the strain-rate conditions.

More recently, the effects of unsteady strain rate have also been studied for hydrogen/air [6] and methane/air [7] mixtures, considering a monochromatic sinusoidal strain-rate oscillation. Sung and Law [6] found that an initially nonignitable system may ignite under oscillatory conditions if the excursion time over favorable strain conditions is long enough compared with a characteristic ignition delay time. Consistent results were found by Mason et al. [8], who studied the effects of impulsive strain-rate forcing on the ignition of nonpremixed hydrogen/air mixtures.

The preceding results from unsteady ignition studies seem to suggest that the ultimate fate of an ignition kernel depends on the history of the temporal excursion of the strain rate during the ignition delay. In our recent study [9], however, the effect of temperature fluctuations on the ignition of a homogeneous mixture was found to be twofold: whereas the cumulative mean temperature dictates the ignition behavior for low-frequency oscillations, as the frequency increases, ignition behavior depends more strongly on the instantaneous phasing of the unsteady fluctuation. This result implies that the ignition behavior in a one-dimensional unsteady system may be more complex than might be expected from the previous studies.

Therefore, in this paper, we revisit the effects of sinusoidal fluctuations in the scalar dissipation rate on the autoignition behavior of a nonpremixed hydrogen/air system. This investigation was motivated in part by the recent development of the low-temperaturecombustion engines, such as the homogeneous-charge compressionignition engines, in which the start of combustion is determined by autoignition of mixture in the presence of velocity and scalar fluctuations at varying degrees. Therefore, there is a strong interest in the fundamental understanding of the overall effect of turbulence on autoignition for a wide range of parametric conditions. Considering 
the findings from previous studies, we extend the parametric studies to consider a wider range of frequencies and different mean scalar dissipation rates with respect to the steady ignition limit. Detailed investigation is undertaken to examine the ignition-kernel growth. A nondimensional ignitability parameter is proposed based on the ignition-kernel Damköhler number, such that the unsteady ignition delay behavior can be uniquely mapped to this parameter. Subsequently, a unified ignitability criterion is proposed.

\section{Formulation and Numerical Method}

The computational configuration is a counterflow mixing layer between two opposing axisymmetric nozzles separated by a fixed distance, as shown in Fig. 1. The conservation equations for this configuration can be found in previous work [7,10], in which a compressible formulation was used to capture fast transients associated with ignition. The governing equations are solved using OPUS [10], which is an opposed-flow solver using a onedimensional similarity coordinate. The code employs variable-order implicit time integration with adaptive time stepping for robust handling of numerical stiffness [11]. The code is interfaced with CHEMKIN [12] and Transport [13] packages for computing detailed reaction rates and transport properties.

A detailed hydrogen mechanism with 19 reactions and 9 species [14] is used in this study. For all of the results presented in this study, $\overline{\mathrm{H}_{2}}(50 \%)$ diluted with $\mathrm{N}_{2}(50 \%)$ is supplied from one nozzle, impinging against air $\left(79 \% \mathrm{~N}_{2}\right.$ and $\left.21 \% \mathrm{O}_{2}\right)$ stream from the other nozzle $0.5 \mathrm{~cm}$ apart. The pressure is fixed to $2 \mathrm{~atm}$. Fuel-side temperature is fixed to $300 \mathrm{~K}$ and air-side temperature is fixed to $1020 \mathrm{~K}$. The crossover temperature at this pressure is found to be $985 \mathrm{~K}$, at which the rates of branching and termination reactions are equal [15]. The imposed oxidizer temperature results in the ignitionkernel temperature being higher than the crossover value, and therefore the condition corresponds to that of the high-temperature ignition regime. To study unsteady scalar dissipation rate effects, a time-varying velocity is specified at both nozzle inlets, as given by

$$
V(t)=V_{0}(1+A \sin (2 \pi f t))
$$

where $\left|V_{0}\right|, A$, and $f$ are set to identical values at both nozzle exits. To compute the ignition delay, a nonreactive steady solution is first obtained while suppressing all reaction rates. This generates a converged initial solution for the unsteady computation. The unsteady calculation is then performed with reactions turned on at $t=0$.

\section{Ignition Response to a Steady Scalar Dissipation Rate}

As a reference, the response of the ignition delay to steady scalar dissipation rate is studied. The velocity at the nozzle is fixed in this case. For all of the results presented in this study, the mixture fraction is defined using Bilger's [16] definition as

$$
Z=\frac{Y_{\mathrm{H}} / 2 W_{\mathrm{H}}-\left(Y_{\mathrm{O}}-Y_{\mathrm{O}, \text { air }}\right) / W_{\mathrm{O}}}{Y_{\mathrm{H}, \text { fuel }} / 2 W_{\mathrm{H}}+Y_{\mathrm{O}, \text { air }} / W_{\mathrm{O}}}
$$

and the scalar dissipation rate is defined as

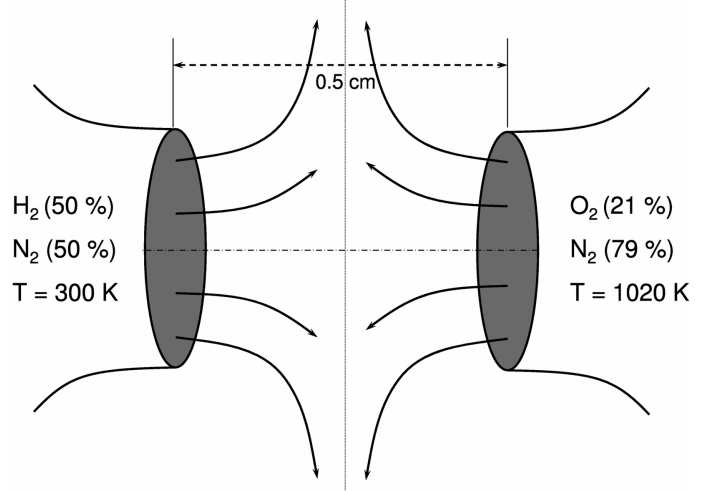

Fig. 1 Computational model configuration.

$$
\chi=2 D\left(\frac{\partial Z}{\partial x}\right)^{2}
$$

where $D$ is the thermal diffusivity. Unless stated otherwise, the scalar dissipation rate is determined at the ignition kernel, which is defined as the location of the maximum heat release rate. The onset of ignition is defined as the instant at which the rate of change of the peak temperature in the domain becomes maximum.

Figure 2 shows the ignition delay and maximum steady-state $\mathrm{OH}$ mass fraction in the domain as a function of the nozzle inlet velocity. The solid line in the maximum $Y_{\mathrm{OH}}$ plot represents the computed lower branch of the standard $S$-curve of the flame response. The dashed line in the same plot shows the hand-drawn continuation of the lower branch to unstable and fully burning branches. The inlet velocity gives a measure of the scalar dissipation rate determined at the ignition kernel. As the inlet velocity is decreased, the frozen steady-state solution reaches the steady turning point near the velocity at $1155 \mathrm{~cm} / \mathrm{s}$. At this steady ignition limit, the corresponding scalar dissipation rate at the ignition kernel is found to be $\chi_{s}^{*}=47 \mathrm{~s}^{-1}$, where subscript $s$ denotes the steady limit. The mixing layer is ignitable for $\chi<\chi_{s}^{*}$. If this condition is met, the ignition delay gradually increases as $\chi$ increases as a result of increased loss of radicals and heat from the ignition kernel. Note that the ignition delay plotted on the left axis is obtained from the unsteady computation, whereas the maximum $\mathrm{OH}$ mass fraction plotted on the right axis is obtained from steady computation. These ignition results under the steady scalar dissipation rate conditions will be used to explain the results for the unsteady scalar dissipation rate cases presented in the next section.

\section{Ignition Response to Unsteady Scalar Dissipation Rate}

The response of the ignition delay to the unsteady scalar dissipation rate is now examined. The initial value $\left(V_{0}\right)$ of the velocity oscillation at the nozzle inlet is fixed at $1050 \mathrm{~cm} / \mathrm{s}$. Two different amplitudes of velocity oscillation are considered: $A=0.4$ (case A) and 0.8 (case B). Because $V_{0}$ is close to the steady ignition limit, the nozzle velocity for both cases becomes significantly greater than the steady ignition limit during the oscillatory cycle. The main consideration is the effect of frequency of oscillation on the overall ignition delay.

Figures $3 \mathrm{a}$ and $3 \mathrm{~b}$ show the temporal variation in $\chi$ for cases $\mathrm{A}$ and $\mathrm{B}$, respectively, for various frequencies of oscillation. It is seen that $\chi$ responds sinusoidally, whereas its amplitude of oscillation becomes attenuated as the frequency increases. In both figures, the steady ignition limit $\chi_{s}^{*}$ is also plotted as a bold line. It appears that the ultimate fate of the mixture (whether it ignites or not) strongly depends on the overall mean scalar dissipation rate $\bar{\chi}$ during the induction period. A rational definition of the mean scalar dissipation

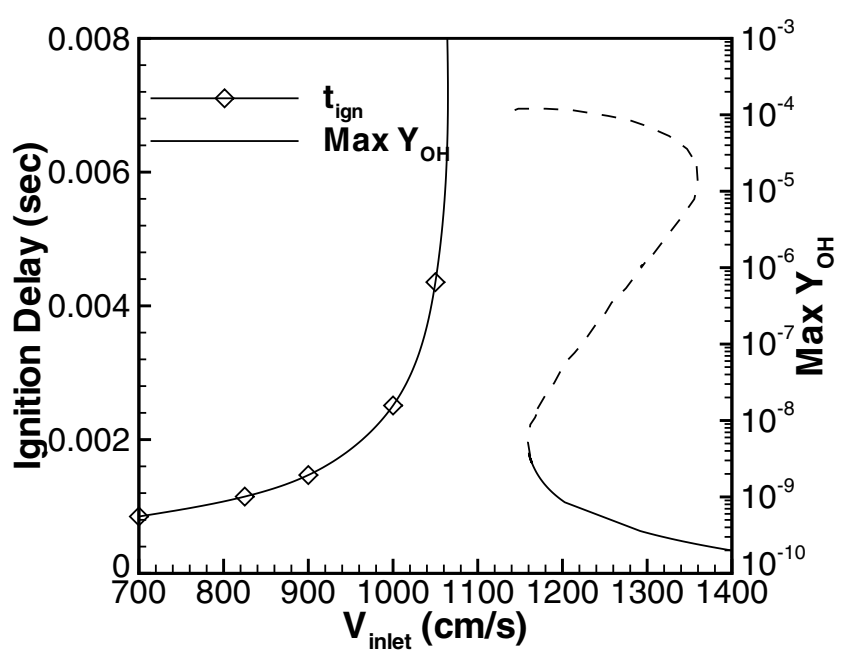

Fig. 2 Ignition delay and maximum $Y_{\mathrm{OH}}$ as a function of velocity at nozzle inlet. 
rate is given by

$$
\bar{\chi}=\frac{1}{t_{\text {ign }}} \int_{0}^{t_{\mathrm{ign}}} \chi \mathrm{d} t
$$

where $t_{\text {ign }}$ is the ignition delay for each frequency condition. For low frequencies, $\bar{\chi}$ depends on the initial phasing of the oscillation, whereas it converges to an asymptotic value at higher frequencies as a large number of oscillatory cycles occur during the induction period.

Comparing Figs. $3 \mathrm{a}$ and $3 \mathrm{~b}$, it is evident that the system in case $\mathrm{A}$ ignites for all frequencies, as $\bar{\chi}$ always falls below $\chi_{s}^{*}$. For case B, however, $\bar{\chi}$ exceeds $\chi_{s}^{*}$ at high frequencies and hence the system fails to ignite.

Figures $4 \mathrm{a}$ and $4 \mathrm{~b}$ show the ignition delay and $\bar{\chi}$ as a function of frequency for cases $\mathrm{A}$ and $\mathrm{B}$, respectively. For case A, the ignition characteristics are categorized into three regimes. At low frequencies (regime I), ignition occurs before one complete cycle of oscillation. In this case, the ignition behavior is characterized as a quasi-steady response, such that the ignition delay is dictated by the mean scalar dissipation rate during the induction time. This is clearly confirmed by the strong correlation between the ignition delay and $\bar{\chi}$. At intermediate frequencies (regime II), the ignition delay increases with frequency, whereas $\bar{\chi}$ remains almost constant. In this regime, ignition depends on the detailed unsteady response of the ignition kernel throughout the induction period. This issue will be further investigated in the next section.

Finally, at higher frequencies (regime III), the ignition kernel no longer responds to the rapid oscillation and the system recovers the quasi-steady characteristics. As in regime I, whether the mixture

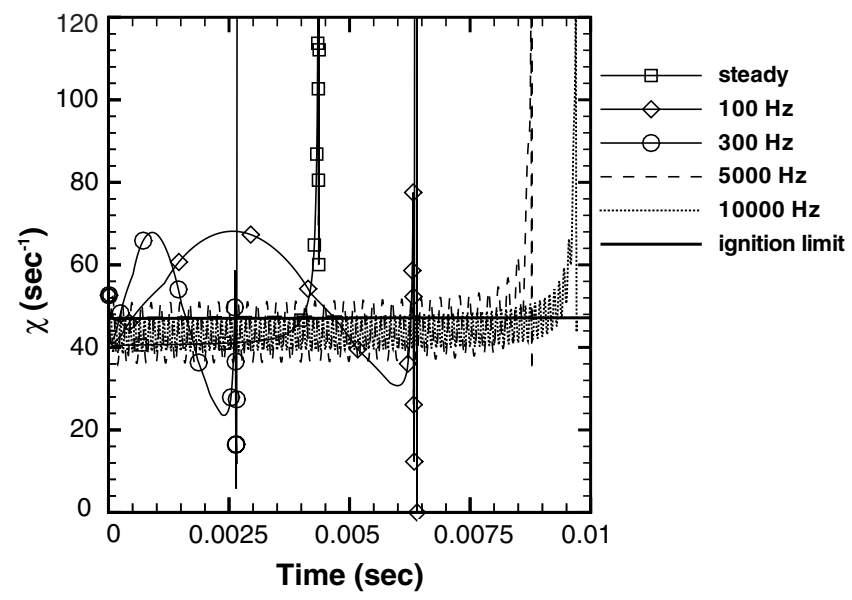

a) Case $\mathrm{A}$

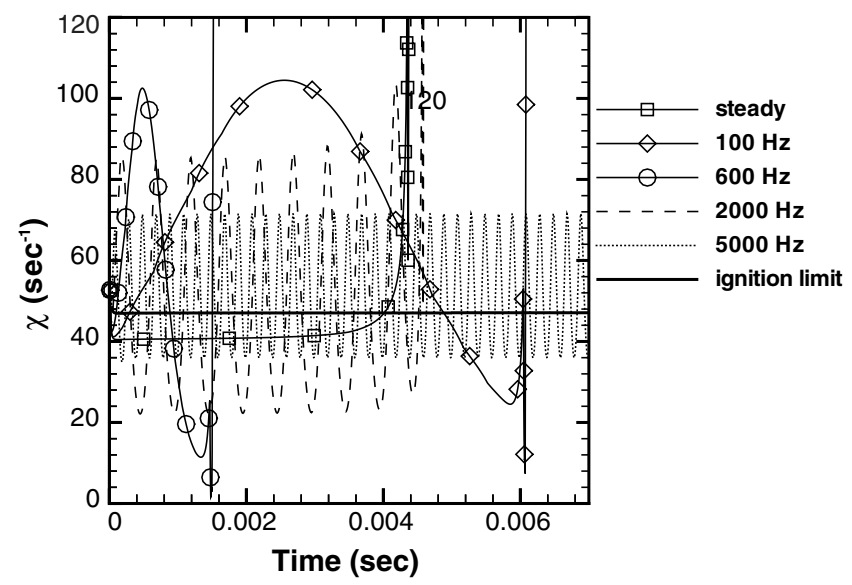

b) Case B

Fig. 3 Scalar dissipation rate at the ignition kernel as a function of time for different frequencies of velocity oscillation at the nozzle inlet for a) case $A$ and b) case $B$.

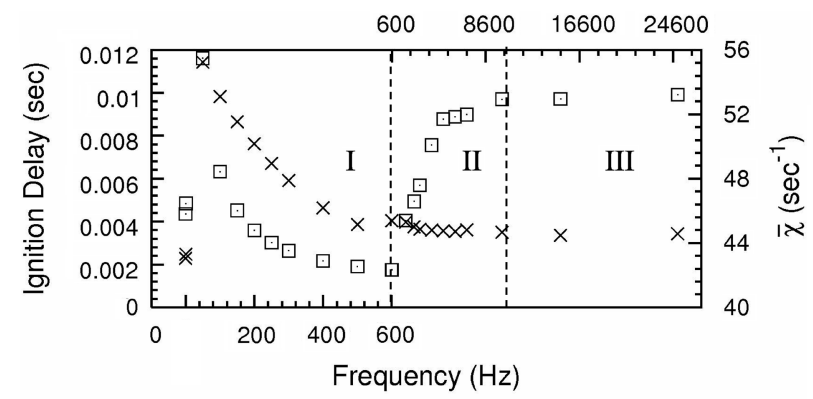

a) Case $A$

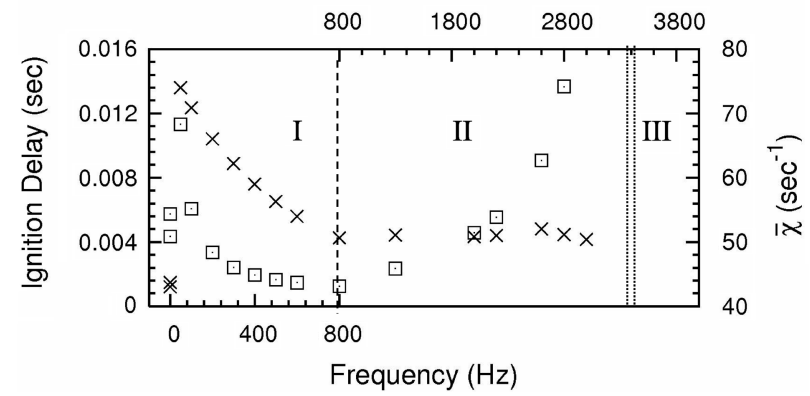

b) Case B

Fig. 4 Ignition delay $(\square)$ and $\bar{\chi}(x)$ as a function of frequency for a) case $A$ and $b$ ) case $B$. Note that the frequency scale is split between the bottom and top axes.

ignites or not again depends entirely on the mean scalar dissipation rate relative to the steady limit. For case A, ignition occurs because $\bar{\chi}<\chi_{s}^{*}$, and in this quasi-steady regime, it is clearly seen that both ignition delay and $\bar{\chi}$ remain almost constant over a wide range of frequencies. Figure $4 \mathrm{~b}$ shows the results for case B, for which $\bar{\chi}$ exceeds $\chi_{s}^{*}$ in the high-frequency limit. As a result, all of the trends are similar to those shown in Fig. 4a, except that ignition does not occur in the high-frequency limit. Therefore, it is difficult to identify the boundary between regime II and regime III in case $\mathrm{B}$.

Note that for the chosen amplitudes of oscillation $(A=0.4$ and 0.8 for cases $\mathrm{A}$ and $\mathrm{B}$, respectively), the ignition delay in regime I first increases and then decreases for both cases. However, if the phase of oscillation was shifted by $180 \mathrm{deg}$, such that scalar dissipation rate first decreases and then increases, then the ignition delay would first decrease as frequency increases in regime I. Nonetheless, the quasisteady concept is still valid in that the ignition delay correlates strongly with the mean scalar dissipation rate during the induction period.

The transition from quasi-steady (regime I) to unsteady (regime II) ignition response depends on the ratio of the characteristic chemical time (the ignition delay) to the characteristic time scale of unsteady fluctuation. To confirm this point, the ignition delay shown in Figs. $4 \mathrm{a}$ and $4 \mathrm{~b}$ is plotted against the normalized frequency $f \cdot t_{\text {ign }}$. The normalized frequency represents the number of cycles in $\chi$ oscillation before ignition. Figure 5 shows the results. The results for the two cases collapse very well, showing an initial abrupt rise and decay in the ignition delay. This behavior is consistent with the results in our previous study on the ignition of homogeneous mixture subjected to unsteady temperature fluctuations [9], in which a strong correlation between the ignition delay and the mean temperature was found at low frequencies.

The minimum ignition delay is achieved at approximately $f \cdot t_{\text {ign }}=1$, which is considered to be the boundary between regimes I and II. When the ignition kernel is exposed to more than one cycle of $\chi$ oscillation, the evolution of radical species in the ignition kernel undergoes excursions of loss and growth, and the ignition event has a cumulative effect throughout the induction period. Thus, the ignition response is no longer considered to be quasi-steady and the ignition delay does not correlate with $\bar{\chi}$. To describe the unsteady ignition behavior in the intermediatefrequency range, the temporal history of the Damköhler number at the ignition kernel needs to be considered. 


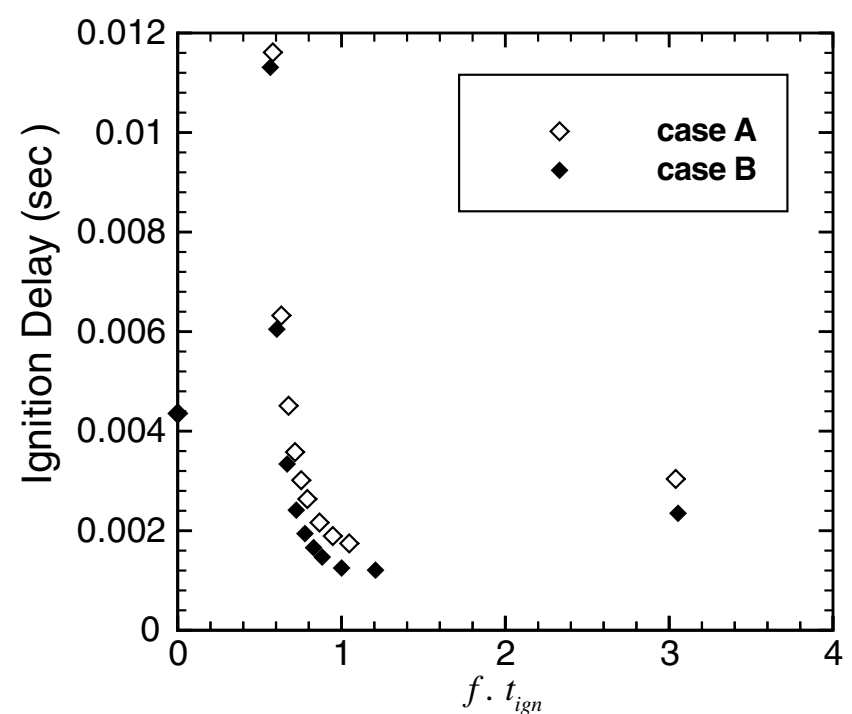

Fig. 5 Ignition delay as a function of frequency times ignition delay for case $A$ and case $B$.

\section{Damköhler Number Response to Oscillatory $\chi$}

Following a previous study [8], the kernel Damköhler number $D a_{\mathrm{H}}$ is defined based on the hydrogen radical as

$$
D a_{\mathrm{H}}=\left|\frac{\dot{\omega}_{\mathrm{H}}}{\rho u\left(\partial Y_{\mathrm{H}} / \partial x\right)+\partial\left(\rho Y_{\mathrm{H}} V_{\mathrm{H}}^{D}\right) / \partial x}\right|
$$

where $V_{\mathrm{H}}^{D}$ is the diffusion velocity of $\mathrm{H}$ radicals. As defined, $D a_{\mathrm{H}}$ at the ignition kernel represents the ratio of chemical production $\dot{\omega}_{\mathrm{H}}$ to the transport loss in the $\mathrm{H}$ radical. Therefore, $D a_{\mathrm{H}}>1$ indicates that the ignition kernel is at a favorable condition to ignite.

Figures 6a and 6b show the temporal history of $D a_{\mathrm{H}}$ for a few representative frequencies in the intermediate-frequency range for cases A and B, respectively. For case B, a high frequency of $5000 \mathrm{~Hz}$, for which no ignition occurs, is also shown. It is seen that $D a_{\mathrm{H}}$ responds to the sinusoidal fluctuations in $\chi$. Note that ignition can occur after a number of oscillations, even if $D a_{\mathrm{H}}<1$ for a significant fraction of the induction period.

An alternative view of Fig. 6b is shown in Fig. 7, in which $D a_{\mathrm{H}}$ and $Y_{\mathrm{H}}$ are plotted in the phase space for three different frequencies. For $f=1600 \mathrm{~Hz}$, as $D a_{\mathrm{H}}$ oscillates around unity, $Y_{\mathrm{H}}$ gradually increases in time and eventually takes off. On the other hand, at higher frequencies $(f=3000$ and $5000 \mathrm{~Hz})$, the evolution in $Y_{\mathrm{H}}$ reaches a limit cycle and no ignition occurs.

These results suggest that the ignition kernel can survive some momentary unfavorable conditions and eventually ignite, depending on the cumulative history of the kernel Damköhler number. It is conjectured that ignition can occur if the favorable condition $\left(D a_{\mathrm{H}}>1\right)$ is maintained for a duration longer than a critical value. To derive a rational criterion, we first determine the fraction of the time duration for which $D a_{\mathrm{H}}>1$ during the ignition delay, given by

$$
\Delta_{t}=\frac{\operatorname{time}\left(D a_{\mathrm{H}} \geq 1\right)}{t_{\mathrm{ign}}}
$$

In other words, $\Delta_{t}$ represents the fractional duration of favorable conditions for ignition. For the cases in which ignition does not occur, we replace the denominator in Eq. (6) by a sufficiently long time such that $\Delta_{t}$ converges. Subsequently, the mean kernel Damköhler number is defined similarly to Eq. (4) as

$$
\overline{D a}_{\mathrm{H}}=\frac{1}{t_{\text {ign }}} \int_{0}^{t_{\text {ign }}} D a_{\mathrm{H}} \mathrm{d} t
$$

Combining Eqs. (ㅁ) and (7), the ignitability $\Gamma$ is defined as

$$
\Gamma=\overline{D a}_{\mathrm{H}} \cdot \Delta_{t}
$$

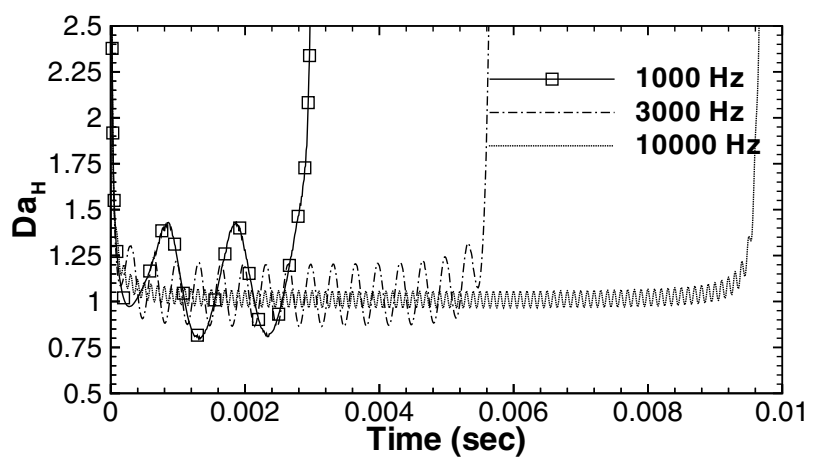

a) Case $A$

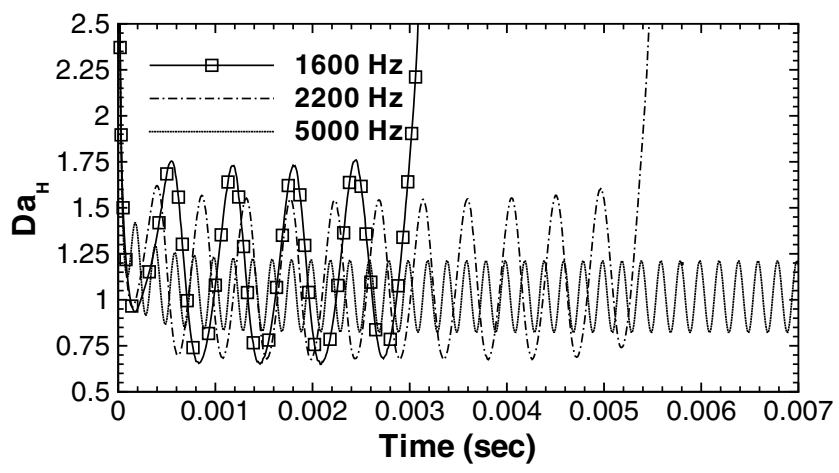

b) Case B

Fig. 6 Temporal history of Damköhler number for different frequencies in the intermediate-frequency range for a) case $A$ and b) case $B$.

The ignitability parameter essentially accounts for the mean value of the kernel Damköhler number during the induction period as well as the fractional duration of the favorable condition for ignition. Figure 8 shows the plot of ignition delay as a function of $\Gamma$ for cases $A$ and $\mathrm{B}$ (symbols) as well as for three additional cases ( $\mathrm{C}$ to $\mathrm{E}$ ) with different values of initial velocity and amplitude $\left[V_{0}, A\right]=$ $[1050,0.7],[1050,-0.8]$, and $[1100,0.7]$, respectively. The negative amplitude implies that the phase of velocity oscillation is shifted by $180 \mathrm{deg}$. Cases C, D, and E have all converged to $\bar{\chi}>\chi_{s}^{*}$ in the highfrequency limit, similarly to case B. Each data point in a given curve represents a result for a different frequency in regime II. Because $\bar{\chi}$

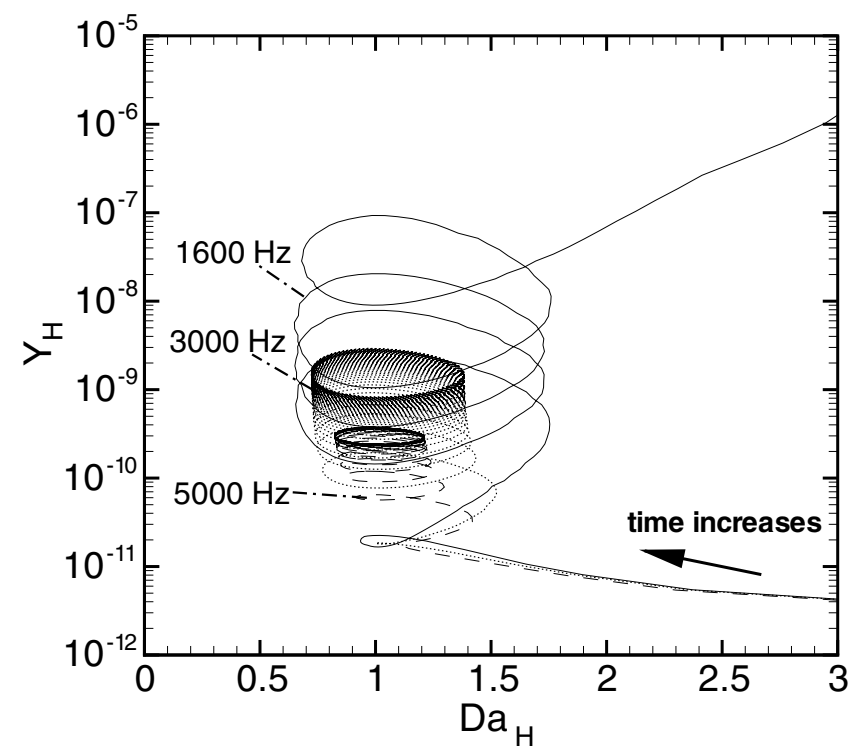

Fig. 7 Transient evolution of $D a_{\mathrm{H}}$ and $Y_{\mathrm{H}}$ as the ignition event takes place, at various frequencies for case $B$. 


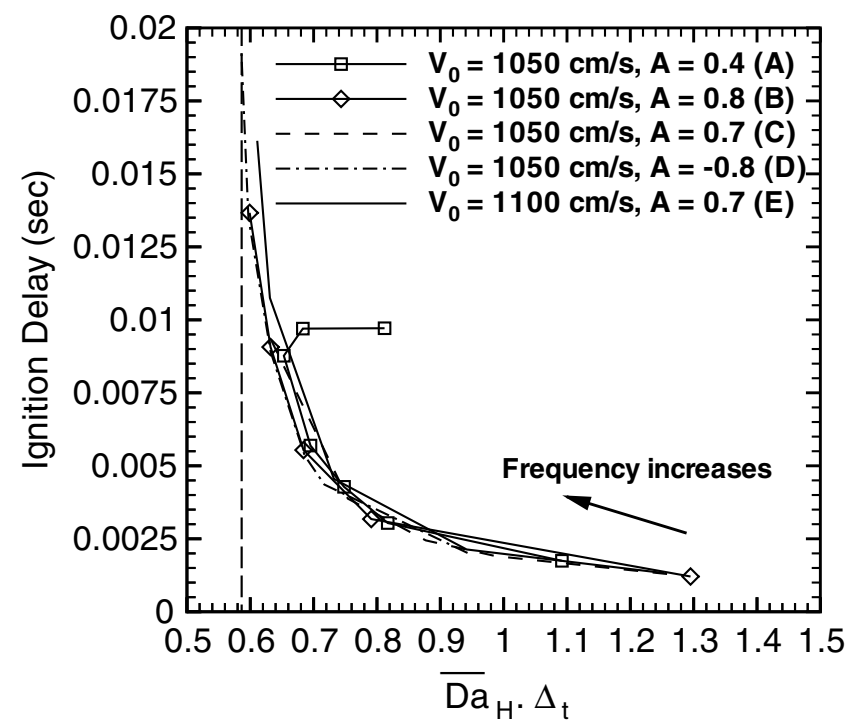

Fig. 8 Ignition delay as a function of ignitability for cases A-E with different frequencies.

differs for different cases, the range of frequency that corresponds to regime II also differs. For example, the frequency range shown in the figure ranges from $1000-2800 \mathrm{~Hz}$ for case $\mathrm{B}$, whereas the range changes to $1300-2900 \mathrm{~Hz}$ for case D. Nevertheless, the ignition delay versus $\Gamma$-curves for all five cases collapse surprisingly well. The only exception is for case A, which has a different highfrequency limit $\left(\bar{\chi}<\chi_{s}^{*}\right)$. In this case, the ignition delay levels off to a constant value in the high-frequency limit (the data shown are for frequencies up to $10,000 \mathrm{~Hz}$ ). As discussed before, at such high frequencies, the ignition response becomes quasi-steady again and thus depends solely on either $\overline{D a}_{\mathrm{H}}$ or $\bar{\chi}$. Note that for all of the conditions considered, no ignition was observed for $\Gamma$ less than 0.59 , which is denoted by a vertical dashed line in Fig. $\underline{8}$. Therefore, the $\Gamma$ parameter proposed in this study provides a generalized ignitability criterion for a wide range of parametric conditions.

\section{Conclusions}

The effects of unsteady scalar dissipation rate fluctuation on the ignition of nonpremixed hydrogen/air were studied using a counterflow configuration. Axial velocity at the nozzle inlet was imposed as a sinusoidal function in time, and the corresponding variation in the scalar dissipation rate at the ignition kernel was adopted as the main parameter. Two cases with different mean scalar dissipation rates were considered based on whether the mean scalar dissipation rate at ignition kernel is less than (case A) or greater than (case B) the steady ignition limit. The results showed that the ignition behavior is characterized in three distinct regimes, depending on the frequency. At low frequencies, such that ignition occurs within one cycle of imposed oscillation (regime I), the ignition delay correlates strongly with the mean scalar dissipation rate during the induction period. At very high frequencies (regime III), the system again recovers a quasi-steady behavior, in which case the ultimate fate of the ignition kernel is dictated by the magnitude of the mean scalar dissipation rate relative to the steady ignition limit.

At intermediate frequencies (regime II), accurate prediction of the ignition delay requires the knowledge of the cumulative history of the unsteady fluctuations. A new criterion for the ignitability, $\Gamma$, was defined as a product of the mean kernel Damköhler number and the fractional duration of favorable conditions for ignition. The ignition delay versus $\Gamma$ showed excellent collapse for a wide range of parametric conditions, demonstrating the validity of the criterion in predicting the unsteady ignition characteristics. It was found that the ignition delay increases as $\Gamma$ decreases, and the critical value of $\Gamma$ was identified, below which the kernel fails to ignite because the radical generation cannot overcome the increased transport losses throughout the oscillatory cycles.

\section{Acknowledgments}

This study was supported by the University Consortium on LowTemperature Combustion for High-Efficiency, Ultra-Low Emission Engines, directed by the University of Michigan, and funded by the U.S. Department of Energy. Su-Ryong Lee would like to thank the financial support by Seoul National University of Technology.

\section{References}

[1] Thevenin, D., and Candel, S., "Diffusion and Premixed Flame Ignition Dynamics in a Field of Variable Strain Rate," Proceedings of the Combustion Institute, Vol. 25, No. 1, 1994, pp. 1547-1554. doi:10.1016/S0082-0784(06)80799-X

[2] Balakrishnan, G., Smooke, M. D., Williams, F. A., "A Numerical Investigation of Extinction and Ignition Limits in Laminar Nonpremixed Counterflowing Hydrogen-Air Streams for Both Elementary and Reduced Chemistry," Combustion and Flame, Vol. 102, No. 3, 1995, pp. 329-340. doi:10.1016/0010-2180(95)00031-Z

[3] Sánchez, A. L., Balakrishnan, G., Liñán, A., and Williams, F. A., "Relationships Between Bifurcation and Numerical Analyses for Ignition of Hydrogen Air Diffusion Flames," Combustion and Flame, Vol. 105, No. 4, 1996, pp. 569-590. doi:10.1016/0010-2180(95)00241-3

[4] Fotache, C. G., Kreutz, T. G., and Law, C. K., "Ignition of HydrogenEnriched Methane by Heated Air," Combustion and Flame, Vol. 110, No. 4, 1997, pp. 429-440. doi:10.1016/S0010-2180(97)00084-9

[5] Kreutz, T. G., and Law, C. K., "Ignition in Nonpremixed Counterflowing Hydrogen Versus Heated Air: Computational Study with Detailed Chemistry," Combustion and Flame, Vol. 104, No. 1-2, 1996, pp. $157-175$. doi:10.1016/0010-2180(95)00121-2

[6] Sung, C. J., and Law, C. K., "Ignition of Oscillatory Counterflowing Nonpremixed Hydrogen Against Heated Air," Combustion Science and Technology, Vol. 129, 1997, pp. 347-370. doi: $10.1080 / 00102209708935732$

[7] Im, H. G., Raja, L. L., Kee, R. J., and Petzold, L. R., "A Numerical Study of Transient Ignition in Counterflow Nonpremixed Methane-Air Flame Using Adaptive Time Integration," Combustion Science and Technology, Vol. 158, No. 1, 2000, pp. 341-363. doi: $10.1080 / 00102200008947340$

[8] Mason, S. D., Chen, J. H., and Im, H. G., "Effects of Unsteady Scalar Dissipation Rate on Ignition of Non-Premixed Hydrogen/Air Mixtures in Counterflow," Proceedings of the Combustion Institute, Vol. 29, No. 2, 2002, pp. 1629-1636. doi:10.1016/S1540-7489(02)80200-0

[9] Bansal, G., Im, H. G., and Lee, S. R., "Auto-Ignition of Homogeneous Hydrogen/Air Mixture Subjected to Unsteady Temperature Fluctuations," Combustion Theory and Modeling (to be published).

[10] Im, H. G., Raja, L. L., Kee, R. J., Lutz, A. E., and Petzold, L. R., "OPUS: A FORTRAN Program for Unsteady Opposed-Flowed Flames," Sandia National Labs., Rept. SAND2000-8211, Albuquerque, NM, 2000.

[11] Li, S., and Petzold, L. R., "Design of New DASPK for Sensitivity Analysis," Univ. of California, Santa Barbara, Computer Science Dept., Rept. TRCS99-23, Santa Barbara, CA, 1999.

[12] Kee, R. J., Rupley, F. M., and Miller, J. A., "CHEMKIN-II, A FORTRAN Chemical Kinetics Package for the Analysis of Gas-Phase Chemical Kinetics," Sandia National Labs., Rept. SAND89-8009B, Albuquerque, NM, 1991.

[13] Kee, R. J., Dixon-Lewis, G., Warnatz, J., Coltrin, M. E., and Miller, J. A., "A FORTRAN Computer Code Package for the Evaluation of GasPhase Multicomponent Transport Properties," Sandia National Labs., Rept. SAND86-8246, Albuquerque, NM, 1986.

[14] Yetter, R. A., Dryer, F. L., and Rabitz, H., "A Comprehensive Reaction Mechanism for Carbon Monoxide/Hydrogen/Oxygen Kinetics," Combustion Science and Technology, Vol. 79, 1991, pp. 97-128. doi:10.1080/00102209108951759

[15] Law, C. K., Combustion Physics, Cambridge Univ. Press, New York, 2006.

[16] Bilger, R. W., "The Structure of Turbulent Nonpremixed Flames," Proceedings of the Combustion Institute, Vol. 22, No. 1, 1989, pp. 475488.

doi: $10.1016 / \mathrm{S} 0082-0784(89) 80054-2$ 